\title{
Pemphigus vulgaris macroscopically and cytologically resembling oral squamous cell carcinoma*
}

\author{
Kayo Kuyama ${ }^{1,2}$, Yan Sun ${ }^{1,2}$, Hiroyasu Endo ${ }^{3}$, Etsuko Kaneda ${ }^{4}$, Miyuki Morikawa ${ }^{1,2}$, \\ Masanobu Wakami ${ }^{5}$, Takanori Ito $^{3}$, Hirotsugu Yamamoto ${ }^{1,2}$ \\ ${ }^{1}$ Department of Oral Pathology, School of Dentistry at Matsudo, Nihon University, Matsudo City, Japan \\ ${ }^{2}$ Department of Diagnostic Pathology, School of Dentistry at Matsudo, Hospital of Nihon University, Matsudo City, Japan \\ ${ }^{3}$ Department of Oral Diagnosis, School of Dentistry at Matsudo, Nihon University, Matsudo City, Japan \\ ${ }^{4}$ Department of Pathology, Nihon University School of Dentistry, Chiyoda-Ku, Japan \\ ${ }^{5}$ Department of Crown and Bridge Prosthodontics, School of Dentistry at Matsudo, Nihon University, Matsudo City, Japan \\ Email: kuyama.kayo@nihon-u.ac.jp
}

Received 3 February 2012; revised 4 March 2012; accepted 10 March 2012

\begin{abstract}
We describe the clinical, macroscopic, cytological, histopathological, immunohistochemical, serodiagnostic and aspects of pemphigus vulgaris (PV) in the oral gingiva that clinically mimicked oral squamous cell carcinoma (OSCC) in a 57-year-old Japanese man. He developed slight haphalgesia of the buccal gingiva around teeth numbers 18 and 192 years ago. A dentist diagnosed intractable ulcer, but the patient ignored the condition for about 2 years until a sharp pain in the gingiva worsened. He consulted an otolaryngologist, who referred the patient to our hospital under a cytological diagnosis of OSCC. An oral examination revealed several extensive painful erosions/ ulcers from the buccal and lingual gingiva around teeth numbers 18 to 21 to the distal alveolar mucosa of no. 18 and the buccal and lingual gingiva around tooth number 31. A presumptive diagnosis of $P V$ with dysplastic changes was determined from cytological smears. The cytological Nikolsky test was positive. The diagnosis of PV was confirmed from clinical and histopathological findings of a biopsy specimen obtained from the perilesional site. Although the definitive diagnosis of $P V$ required only 2 weeks after this patient presented at our hospital, 2 years had elapsed since the onset of oral lesions.
\end{abstract}

Keywords: Pemphigus Vulgaris; Oral Squamous Cell Carcinoma; Exfoliative Cytology

\section{INTRODUCTION}

Pemphigus vulgaris (PV) occurs at a rate of 0.5 to 3.2 per 100,000 persons annually [1]. It is a rare autoimmune

\footnotetext{
*A case report of pemphigus vulgaris.
}

blistering disease that can become lethal. It is a vesiculobullous condition that initially presents in the oral mucosa as blisters and erosions/ulcers, followed by lesions involving the skin, and occasionally, other mucosal surfaces. Fatal dehydration or secondary systemic infections can arise in patients with PV if treatment with corticosteroids is delayed [2]. Oral lesions can persist for months or even years before progressing to extraoral sites [3]. Oral PV is still diagnosed after a considerable delay mostly in male patients with a chronic course [4]. Timely recognition and appropriate treatment are important for the prognosis of autoimmune bullous disorders. Differentiation from other bullous and erosive oral diseases is occasionally difficult, especially in desquamative and ulcerated lesions.

The use of exfoliative cytology as a diagnostic tool is controversial, because this technique delays the definitive diagnose of PV [5], although it is valuable for rapidly screening intractable ulcers. Here, we describe various aspects of $\mathrm{PV}$ in the oral gingiva that clinically mimic oral squamous cell carcinoma (OSCC). We believe that these clinical and cytological data are meaningful from the viewpoint of comparative PV and OSCC findings.

\section{CASE REPORT}

A-57-year-old Japanese man developed slight haphalgesia of the buccal gingiva around teeth numbers 18 and 19 2 years ago. A dentist at a private dental clinic simply observed the progress of the symptoms. The patient was dissatisfied with this strategy and consulted an otolaryngologist, who diagnosed intractable stomatitis and prescribed topical corticosteroid. However, the patient did not comply with the medication and about 18 months later, he presented at the otolaryngology department of a certain private University Hospital with worsening sharp 
gingival pain. Squamous cell carcinoma (SCC) was suspected from the findings of exfoliative cytology and he was referred to Nihon University School of Dentistry at Matsudo Hospital.

\subsection{Oral Examination}

An oral examination revealed extensive multiple painful erosions/ulcers from the buccal and lingual gingiva around teeth numbers 18 to 21 to the distal alveolar mucosa of teeth numbers 18 (Figure 1), and the buccal and lingual gingiva around tooth number 31 . The ulcers were hemorrhagic and ablated epithelium had adhered to the surface of the epithelium. He had no skin or extraoral lesions, and a review of his medical history was unremarkable. A differential diagnosis included PV, mucous membrane pemphigoid and OSCC.

\subsection{Oral Exfoliative Cytological Findings}

Cytological smears of buccal gingiva exfoliated using a cytobrush (Medscand Medical AB, Malmo, Sweden) before obtaining biopsy specimens were fixed in $95 \%$ ethyl alcohol, and then visualized using Papanicolaou and Giemsa stain. The smear was obviously hypercellular on a sanguinous background. Parabasal large cells formed loosely cohesive sheets, and round/oval single cells were numerous. The nuclear/cytoplasmic ratio was high, but the nuclear outline was extremely smooth and the chromatin was vesicular with $1-3$ prominent nucleoli (Figure 2). The cytoplasm was dense and sheets were characterized by frayed, wispy cell margins (Figure 3).

The cytological features were similar to those of repair, except for the striking predominance of single cells. However, many atypical keratinized superficial squamous cells were scattered among Tzanck cells. The pumpkin yellow cytoplasm was dense and well keratinized, the chromatin was irregular and vesicular (Figure 2) and

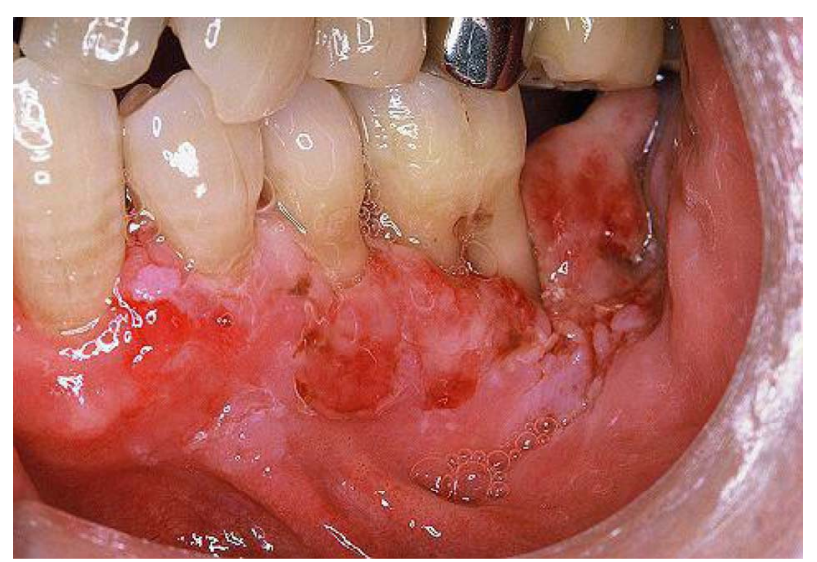

Figure 1. Painful erosion/ulcer from the buccal and lingual of the gingival around No. 18 to 21 to distal alveolar mucosa of No. 18

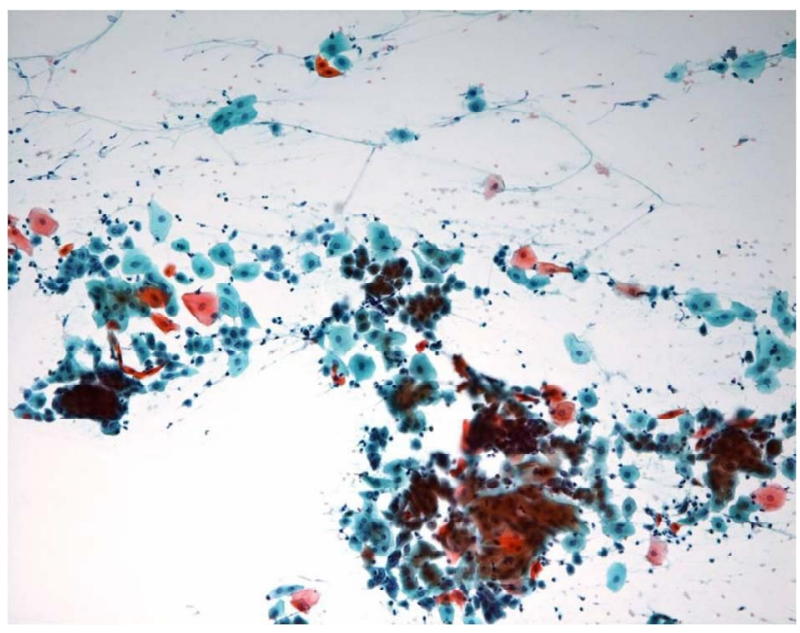

Figure 2. The smear was markedly hypercellular with a very blood-stained background ( $\times 100$, Pap.).

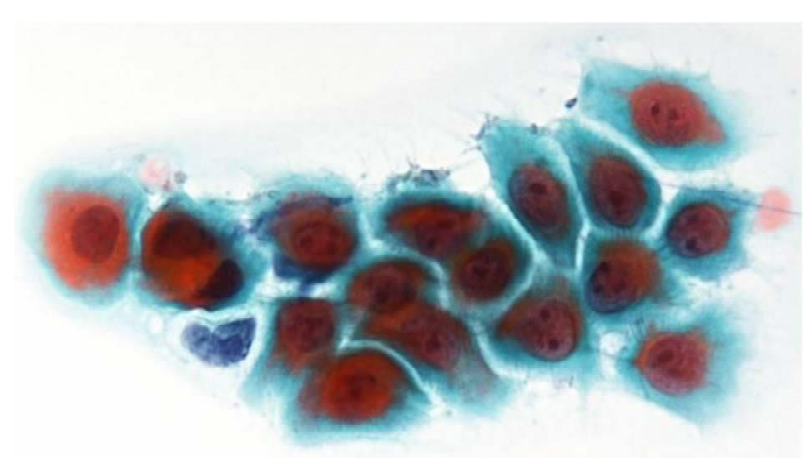

Figure 3. Tzanck cells showed the N/C ratio was high, but the nuclear outline was extremely smooth $(\times 400$, Pap.).

multinuclear atypical cells were found among Tzank cells (Figure 4). These findings indicated a presumptive diagnosis of PV with dysplastic changes.

\subsection{Biopsy Findings}

The results of the cytological Nikolsky test were positive. A gingival biopsy from the perilesional site was examined by routine histopathology and the direct immunofluorescence (DIF) test. The biopsy comprised intramucosal vesicles containing floating, round keratinocytes (Tzanck cells) that had become detached from the surrounding cells (acantholysis). The basal layer cells were still firmly attached to the connective tissue (Figure 5). A keratin pearl formation caused by keratinized, hyperplastic, picnotic and non-dysplastic squamous cells was located adjacent to a blister. The adjacent connective tissue contained mild to moderate chronic inflammatory infiltrates (Figure 6).

Direct immunofluorescence analysis using conjugates for $\operatorname{IgG}, \operatorname{IgA}, \operatorname{IgM}, \mathrm{C} 3$ and fibrinogen revealed $\operatorname{IgG}$ and C3 deposition between the epithelial cells, forming a fishnet appearance (Figure 7). The basement membrane 


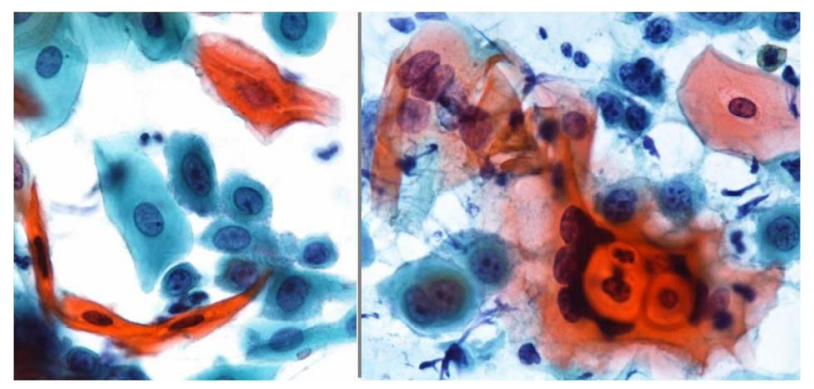

Figure 4. Many atypical kratinized superficial squamous cells were scattered among Tzanck cells $(\times 400$, Pap.).

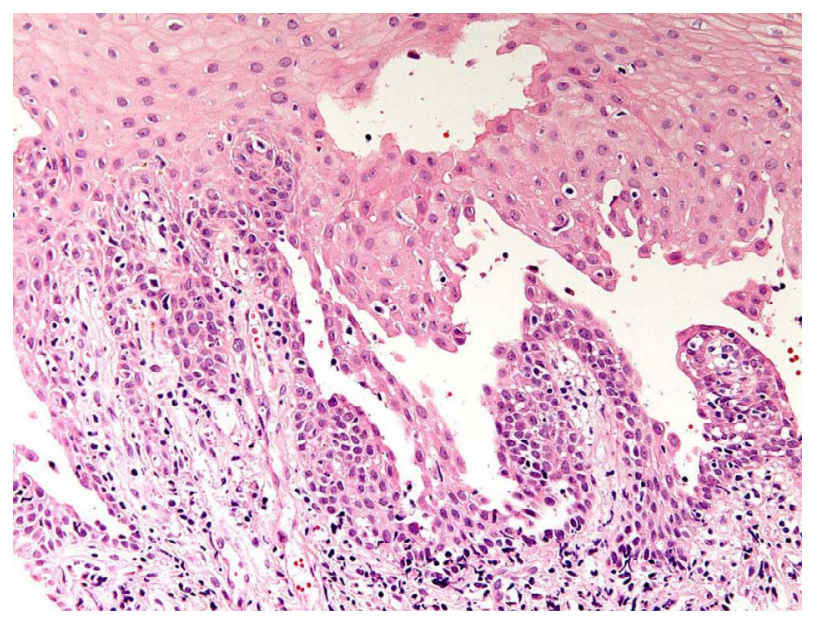

Figure 5. An intramucosal vesicle containing Tzanck cells, and the basal layer cells are still firmly attached to the connective tissue $(\times 200$, H.E. $)$.

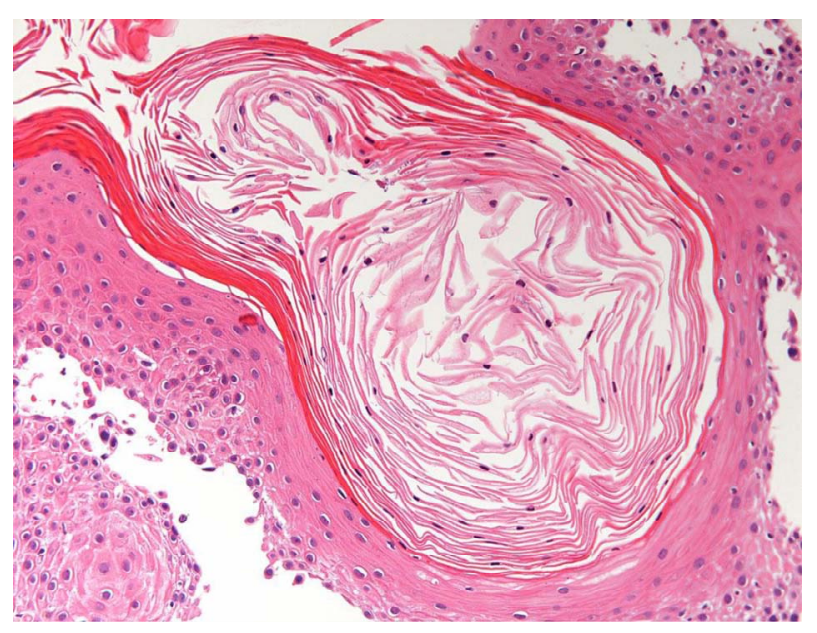

Figure 6. Keratin pearl formation by picnotic squamous cells was observed $(\times 200$, H.E.).

zone was negative for IgG and $\mathrm{C} 3$ deposition. The serodiagnostic indices for Dsg 1 and Dsg 3 were 35 and 133 , respectively.

The clinical and histopathological findings confirmed a diagnosis of PV. Although this patient was diagnosed with PV within 2 weeks of arriving at our hospital, 2

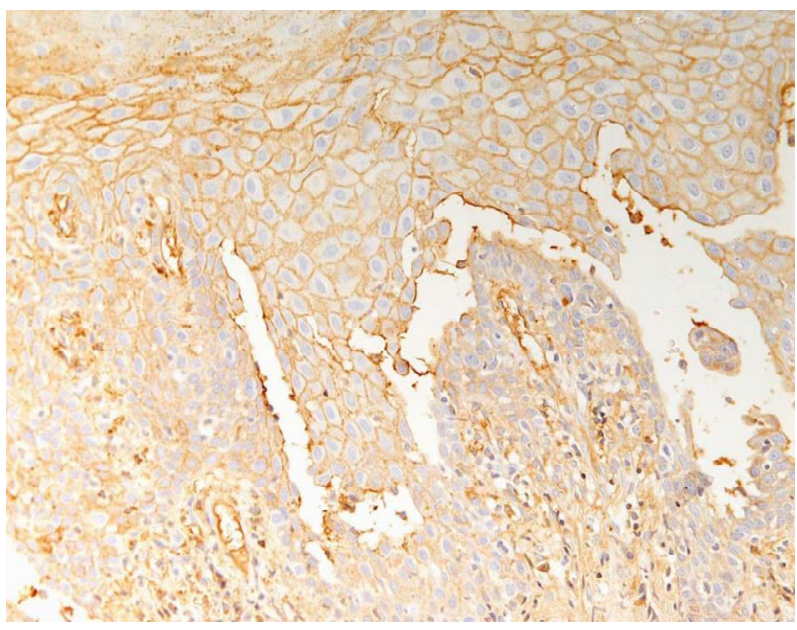

Figure 7. IgG revealed positive reaction between the epithelial cells, forming a "fish net" appearance $(\times 400)$.

years had elapsed since the onset of the oral lesions. The gingival lesions were treated with $0.1 \%$ triamcinolone acetonide. Cytology confirmed that the gingival ulcers had receded and that the Tzanck cells had disappeared at 4 and 6 years later, respectively.

\subsection{Treatment Outcome}

The patient was immediately referred to the dermatologist and the treatment of PV was started using systemic corticosteroid. Additionally, topical corticosteroid $(0.1 \%$ triamcinolone acetonide) was provided for the treatment of gingival PV. Plastic stent was constructed in order to occlude and contain the topical corticosteroid. The patient responded well to topical and systemic therapy. Cytology confirmed that the gingival ulcers had receded and that the Tzanck cells had disappeared at 4 and 6 years later, respectively.

\section{DISCUSSION}

Pemphigus vulgaris is a blistering disease of skin and sometimes of the mucous membranes. The six major types of pemphigus are classified according to anatomic features of lesions and the target antigens recognized by the autoantibodies. Pemphigus vulgaris accounts for $80 \%$ of patients with pemphigus [6], and it comprises mucous membrane-predominant (anti-desmoglein 3 only) and mucocutaneous (anti-desmoglein 1 and 3) immune variants.

Most patients with PV are aged between 50 and 70 years [7], whereas bullous pemphigoid predominantly affects mostly individuals older than 70 years $[7,8]$. About $90 \%$ of patients with PV have skin lesions with oral manifestations $[7,8]$. There is a $40 \%$ to $70 \%$ probability that the initial site of PV involvement is in the oral cavity [2,7-10]. 
Persistent PV ulcers can affect any part of the oral cavity, but they usually appear first in the buccal mucosa, palatal mucosa and lips $[11,12]$. Approximately $50 \%$ of the lesion which suffered from PV was already in trouble for a long period of time [13]. Since the mucosal lining of the oral cavity is very thin, PV is usually discovered as an erosive/ulcerative lesion. Oral ulcers are initially red and surrounded by whitish areas of infection. They develop a yellowish slough and heal slowly, but rarely with scarring. Gingival lesions usually comprise highly desquamative or erosive gingivitis, whereas bullae rupture to leave flaps of peeling tissue with red erosion or deep ulcerative craters mainly on the attached gingiva [14]. The macroscopic findings of our patient were intractable ulcer with an irregular border and deep and necrotic desquamating epithelial tissue fragments adhering to the circumference. These features resembled those of gingival cancer.

Cutaneous lesions are diagnosed within 6 months in $99 \%$ of patients, whereas only $57 \%$ of oral lesions are diagnosed within this period. Furthermore, $70 \%$ of patients see over four specialists before a diagnosis of PV is confirmed [15] and 2 years had elapsed before our patient was correctly diagnosed. In addition, an otolaryngologist misdiagnosed our patient with gingival cancer.
A cytological diagnosis of PV based on mucosal lesions as described by Tzanck [16] is a practical and noninvasive method of demonstrating acantholytic epithelial cells. The cytology of oral PV has been described in detail [17-20], and a presumptive diagnosis can be comparatively simple if characteristic Tzanck cells are evident $[17,18]$.

However, gingival erosive lesions with macroscopic findings similar to those of other conditions require a differential diagnosis among $\mathrm{PV}$, mucous membrane pemphigoid, erosive lichen planus and squamous cell carcinoma; these are summarized in Table 1.

The cytology of PV is typically hypercellular, with many single cells and closely cohesive sheets. The background can be sanguineous, but true tumor diathesis is absent. The cells are parabasal and have a high N/C ratio. The nucleolus, however, has a smooth thin nuclear membrane and very fine, evenly granular, vesicular and transparent chromatin, in contrast to the hyperchromatic coarse chromatin of malignant cells.

Epithelial cells with clear acantholytic changes (Tzanck cells) are evident in smears obtained from $93 \%$ of patients with OPV [21]. Acantholytic cells on cervicovaginal smears may be misdiagnosed as malignant in the absence of a history of pemphigus [22]. Our patient was also estimated by otolaryngology as having OSCC, and

Table 1. The cytological discriminating points of erosive lesion of the oral mucosa.

\begin{tabular}{|c|c|c|c|c|c|}
\hline & \multirow{2}{*}{ Background } & \multicolumn{2}{|l|}{ LPF } & \multicolumn{2}{|c|}{$\mathrm{HPF}$} \\
\hline & & Character of the cells & Distribution pattern & Nuclear & Cytoplasm \\
\hline \multirow{4}{*}{ PV } & \multirow{4}{*}{ Bloody-inflammatory } & \multirow{4}{*}{ Parabasal-sized abnormal cellls } & \multirow{4}{*}{ Sheet-scattered } & $\mathrm{N} / \mathrm{C}$ increasing & Round-oval single cells \\
\hline & & & & Smooth border & Parabasal-sized \\
\hline & & & & 1-3 prominent nucleoli & Two-ton color \\
\hline & & & & Fine chromatin & \\
\hline \multirow{3}{*}{ MMP } & \multirow{3}{*}{ Clear-inflammatory } & \multirow{3}{*}{ Superficial cells } & \multirow{3}{*}{ Scattered } & Nuclear swelling & Clear border \\
\hline & & & & Smooth outline & \\
\hline & & & & Fine chromatin & \\
\hline \multirow{3}{*}{ EL } & \multirow{3}{*}{ Inflammatory (lymphositosis) } & \multirow{3}{*}{ Superficial cells } & \multirow{3}{*}{ Scattered } & Nuclear swelling & Keratinized \\
\hline & & & & Smooth outline & Clear border \\
\hline & & & & Fine chromatin & \\
\hline \multirow{4}{*}{$\mathrm{SCC}$} & \multirow{4}{*}{ Bloody-inflammatory-necrotic } & \multirow{4}{*}{ Superficial-intermediate cells } & \multirow{4}{*}{ Clumping-scattered } & $\mathrm{N} / \mathrm{C}$ increasing & Thick \\
\hline & & & & Irregular border & Irregular border \\
\hline & & & & 1-2 prominent nucleoli & Various sizes \\
\hline & & & & Dense chromatin & Pumpkin colored \\
\hline
\end{tabular}

LPD: Low power field; HPF: High power field; PV: Pemphigus vulgaris; MMP: Mucous membrane phemphigoid; EL: Erosive lichen planus; SCC: Squamous cell carcinoma. 
we considered PV with dysplastic change as he had no history of PV. Cells from PV with atypical squamous changes frequently match the criteria for malignancy [23]. Additionally, immunohistochemistry shows the loss of intercellular epidermal antigens oral SCC and oral epithelial dysplasia [24].

The cell population in smears from acantholytic lesions consists of degenerating superficial squamous cells; nucleoli are prominent, and Tzanck cells are present. The appearance of atypical keratinized superficial squamous cells in our patient indicated the degeneration of exfoliated epithelium, or a reparative keratin pearl origin. The cytological features resembled those of dysplasia and/or repair, but many more single cells were also present. A further diagnostic consideration is the loose cohesion of sheets with a wispy peripheral cytoplasmic border [25]. Acantholysis due to supra-basal and spinous cell degeneration is characteristic of PV, pemphigus foliaceus, familial benign pemphigus and Darier's disease.

Although the cytological findings could indicate a presumptive diagnosis, confirmation was needed for our patient. A diagnosis of PV can be confirmed based only on biopsy findings of involved mucosae. A detailed immunocytological test can be recommended for a final diagnosis and might even be substituted for the histological and direct immunofluorescence assessment of biopsy samples [21]. However, an esophageal study of PV found that only $18.8 \%$ of biopsy specimens actually include the basement membrane [26].

Biopsy is a surgical procedure, and since the actions of dentists can considerably influence outcomes, the first step to defining erosive/ulcerative lesions of the oral mucosa is to recommend exfoliative cytology.

\section{REFERENCES}

[1] Weinberg, M.A., Insler, M.S. and Campen, R.B. (1997) Mucocutaneous features of autoimmune blistering diseases. Oral Surgery, Oral Medicine, Oral Pathology, Oral Radiology, and Endodontology, 84, 517-534. doi:10.1016/S1079-2104(97)90269-9

[2] Ahmed, A.R. and Moy, R. (1982) Death in pemphigus. Journal of the American Academy of Dermatology, 7, 2228.

[3] Laskaris, G., Vareltzidis, A. and Capetanakis, J. (1978) A clinical study of pemphigus: Observeations on 128 patients. Materia Medica Greca, 6, 627-630.

[4] Scully, C., Paes De Almeida, O., Porter, S.R. and Gilkes, J.J. (1999) Pemphigus vulgaris: The manifestations and long-term management of 55 patients with oral lesions. British Journal of Dermatology, 140, 84-89. doi:10.1046/j.1365-2133.1999.02612.x

[5] Endo, H., Rees, T.D., Kuyama, K., Matsue, M. and Yamamoto, H. (2008) Use of oral exfoliative cytology to diagnose desquamative gingivitis: A pilot study. Quintes- sence International, 39, e152-e161.

[6] Kyriakis, K., Tosca, A., Lehou, J., Hatzis, J., Vareltzidis, A. and Stratigos, J. (1989) A five year retrospective study on pemphigus and pemphigoid. Australasian Journal of Dermatology, 30, 33-36. doi:10.1111/j.1440-0960.1989.tb00405.x

[7] Budimir, J., Mihić, L.L., Situm, M., Bulat, V., Persić, S. and Tomljanović-Veselski, M. (2008) Oral lesions in patients with pemphigus vulgaris and bullous pemphigoid. Acta Clinica Croatica, 47, 13-18.

[8] Mignogna, M.D., Lo Muzio, L. and Bucci, E. (2001) Clinical features of gingival pemphigus vulgaris. Journal of Clinical Periodontology, 28, 489-493. doi:10.1034/j.1600-051x.2001.028005489.x

[9] Shklar, G., Frim, S. and Flynn, E. (1978) Gingival lesions of pemphigus. Journal of Clinical Periodontology, 49, 428-436.

[10] Dvoretsky, P.M., Bonfiglio, T.A., Patten, S.F. and Helmkamp, B.F. (1985) Pemphigus vulgaris and microinvasive squamous-cell carcinoma of the uterine cervix. Acta Cytologica, 29, 403-410.

[11] Scully, C. and Challacombe, S.J. (2002) Pemphigus vulgaris: Update on etiopathogenesis, oral manifestations, and management. Critical Reviews in Oral Biology \& Medicine, 13, 397-408. doi: $10.1177 / 154411130201300504$

[12] Mignogna, M.D., Lo Muzio, L., Galloro, G., Satriano, R.A., Rucco, V. and Bucci, E. (1997) Oral pemphigus: Clinical significance of esophageal involvement: Report of eight cases. Oral Surgery, Oral Medicine, Oral Pathology, Oral Radiology, and Endodontology, 84, 179-184. doi:10.1016/S1079-2104(97)90067-6

[13] López-Jornet, P. and Bermejo-Fenoll, A. (2005) Gingival lesions as a first symptom of pemphigus vulgaris in pregnancy. British Dental Journal, 199, 91-92. doi:10.1038/sj.bdj.4812523

[14] Scully, C. and Porter, S.R. (1997) The clinical spectrum of desquamative gingivitis. Seminars in Cutaneous Medicine and Surgery, 16, 308-313. doi:10.1016/S1085-5629(97)80021-1

[15] Ben Lagha, N., Poulesquen, V., Roujeau, J.C., Alantar, A. and Maman, L. (2005) Pemphigus vulgaris: A case-based update. Journal of the Canadian Dental Association, 71, 667-672.

[16] Tzanck, A. (1948) Le cytodiagnostic immediate en dermatologie. Ann Dermatol Syphil, 8, 205-218.

[17] Takahashi, I., Kobayashi, T.K., Suzuki, H., Nakamura, S. and Tezuka, F. (1998) Coexistence of pemphigus vulgaris and herpes simplex virus infection in oral mucosa diagnosed by cytology, immunohistochemistry, and polymerase chain reaction. Diagnostic Cytopathology, 19, 446-450. doi:10.1002/(SICI)1097-0339(199812)19:6<446::AID-D C8>3.0.CO;2-2

[18] Kobayashi, T.K., Kaneko, C., Sugishima, S., Kusukawa, J. and Kameyama, T. (1999) Scrape cytology of oral pemphigus. Report of a case with immunocytochemistry and light, scanning electron and transmission electron microscopy. Acta Cytologica, 43, 289-294. 


$$
\text { doi:10.1159/000330996 }
$$

[19] Silverman, S. Jr. (1965) The cytology of benign oral lesions. Acta Cytologica, 9, 287-295.

[20] Medak, H., Burlakow, P., McGrew, E.A. and Tiecke, R. (1970) The cytology of vesicular conditions affecting the oral mucosa: Pemphigus vulgaris. Acta Cytologica, 14, 11-21.

[21] Ruocco, V., Coscia-Porrazzi, L. and Pisani, M. (1984) Reliability of cytodiagnosis in oral pemphigus vulgaris. A study of 30 cases. Journal of Dermatology, 11, 535-540.

[22] Libcke, J.H. (1970) The cytology of cervical pemphigus. Acta Cytologica, 14, 42-44.

[23] Takahashi, I., Kobayashi, T.K., Suzuki, H., Nakamura, S. and Tezuka, F. (1998) Coexistence of pemphigus vulgaris and herpes simplex virus infection in oral mucosa diagnosed by cytology, immunohistochemistry, and polymerase chain reaction. Diagnostic Cytopathology, 19, 446-450.
doi:10.1002/(SICI)1097-0339(199812)19:6<446::AID-D C8>3.0.CO;2-2

[24] Bovopoulou, O., Sklavounou, A. and Laskaris, G. (1985) Loss of intercellular substance antigens in oral hyperkeratosis, epithelial dysplasia, and squamous cell carcinoma. Oral Surgery, Oral Medicine, Oral Pathology, 60, 648-654. doi:10.1016/0030-4220(85)90369-X

[25] Wright, C., Pipingas, A., Grayson, W. and Leiman, G. (2000) Pemphigus vulgaris of the uterine cervix revisited: Case report and review of the literature. Diagnostic Cytopathology, 22, 304-307. doi:10.1002/(SICI)1097-0339(200005)22:5<304::AID-D C9>3.0.CO;2-X

[26] Galloro, G., Diamantis, G., Mango, L., Inzirillo, M., Mignogna, M.C., Mignogna, C., De Rosa, G. and Iovino, P. (2007) Technical aspects in endoscopic biopsy of lesions in esophageal pemphigus vulgaris. Digestive and Liver Disease, 39, 363-367. doi:10.1016/j.dld.2006.12.008 